\title{
Empathy and Perceived Stress among College Students
}

\author{
Kanchan Gupta ${ }^{1 *}$, Dr. Kiran NC ${ }^{2}$
}

\section{ABSTRACT}

In the present study, the researchers have attempted to assess the relationship between empathy and perceived stress among college students of Karnataka and West Bengal in India. The data was collected using the Basic Empathy Scale for Adults (BES-A) and Perceived Stress Scale (PSS-10) from a sample of 214 college students, in which 107 were boys and 107 were girls. The study found that there was no significant relationship between their cognitive empathy and perceived stress but a significant relationship between their affective empathy and perceived stress was found and the correlation was found to be positive. The study also found that there was a significant gender difference in empathy among college students and girls had a higher empathy than boys, whereas in case of perceived stress, no significant gender difference was found; although the girls showed higher perceived stress levels than boys but the difference in their perceived stress levels was minimal. The implications of nurturing empathy and reducing perceived stress among the college students are discussed.

\section{Keywords: Empathy, Cognitive empathy, Affective empathy, Perceived stress, College students}

\begin{abstract}
A college is an institution of education or a component part of one. The term 'college' in India is usually reserved for institutes offering high school diplomas at year 12 and those offering a bachelor's degree; some colleges, however, offer PhD-level programmes. Colleges are usually situated in various parts of a state and all of them are affiliated to a regional university. Colleges can either be independent or non-autonomous. There are numerous hundred universities and there are affiliated colleges under each university which is often a large number.
\end{abstract}

A student is primarily a person who is enrolled in a school or other educational institution and who is studying to acquire knowledge, establish careers and achieve easy employment in a specific field. College students are essentially people who are pursuing either an undergraduate or postgraduate degree at a college. It is 3 years for undergraduates except for Engineering (BTech or BE), Pharmacy (B.pharm), B.Sc. Agriculture which is a 4-year degree course, Architecture (B.Arch.) which is a 5-year degree course and Medical (MBBS) which consists of a 4.5-year degree course and a 1-year internship, so it is usually a total of 5.5 years and for postgraduation, the course is generally for 2 years.

\footnotetext{
${ }^{1}$ Research Scholar, Dept. of Psychology, Kristu Jayanti College (Autonomous), India

${ }^{2}$ Assistant Professor, Dept. of Psychology, Kristu Jayanti College (Autonomous), India *Corresponding Author
}

Received: March 13, 2021; Revision Received: April 07, 2021; Accepted: April 25, 2021

(C) 2021, Gupta K. \& Kiran NC; licensee IJIP. This is an Open Access Research distributed under the terms of the Creative Commons Attribution License (www.creativecommons.org/licenses/by/2.0), which permits unrestricted use, distribution, and reproduction in any Medium, provided the original work is properly cited. 


\section{Empathy and Perceived Stress among College Students}

\section{Empathy}

The word 'empathy' has its origin from the Greek word 'empatheia', which means feeling the reactions of other persons and entering their world (Campbell \& Babrow, 2004). Emotion researchers typically characterise empathy as the ability to sense other people's feelings, combined with the ability to imagine what someone else would feel or think. Contemporary scholars have also distinguished between two kinds of empathy: Affective Empathy and Cognitive Empathy.

'Affective empathy' refers to the thoughts and feelings that one gets in reaction to the emotions of others, and it may involve mirroring what the other person thinks, or just feeling stressed when one senses the fear or anxiety of another person. 'Cognitive Empathy' refers to the capacity to perceive and accept other people's emotions. It involves taking the viewpoint of another person. Often referred to as perspective-taking, cognitive empathy lends itself to the notion of putting oneself in the shoes of someone else.

Early empathy researchers (e.g. Dymond, 2004, Kohut 1971) described empathy as a cognitive capacity to learn and interpret knowledge for a deeper understanding of the intellectual condition of another person, involving mental tasks. Later, the affective view, which means the sharing of emotions that are manifested in feelings that are subjectively perceived (Cherniss \& Goleman, 2001; Holm, 2001) was introduced. There are also other researchers that accept the cognitive as well as the affective perspective (e.g. Batson, 1995; Eisenberg, 2002).

Students are encouraged to be empathetic because empathetic students help to develop a positive atmosphere in the classroom; empathy sets students up to deepen connections with their current peers and individuals they meet outside college. These individuals can come from different cultures and different socioeconomic backgrounds in our increasingly globalised world than before, thus requiring better established empathy skills. By engaging cross culturally with their peers, as adolescents develop empathy skills, those skills can move to their community lives. The deeper relationships arising from good empathy abilities have the power to reinforce a community and build trust. The implications of community extend well beyond a classroom's four walls. Empathy trains the students to be their community leaders.

\section{Perceived Stress}

Stress is an unavoidable part of life; unless they learn to handle it properly, it can take a toll on the physical health, mental well-being, and academic success of students. College students encounter stress due to shifts in lifestyles, increased workload, new assignments, and interpersonal relationships. Extreme stress levels can impede the quality of work and lead to poor academic performance and attrition.

The college years is the era of emerging adulthood (18-25 years old). These years are years of transition and discovery, a critical moment in the development of identity (Arnett, 2000). There are different stresses involved in such transition and discovery, such as leaving one's family, making new friends, adapting to a new academic life, dating, deciding on one's major, and coping with financial pressures. Thus, in an entirely new social setting marked by greater independence and less adult control than in their previous years, these students experience a number of stresses (Lefkowitz, 2005), an increased interpersonal conflict, homesickness, a sense of alienation (Buote et al., 2007). 
Stress is defined as an adaptive reaction to an external circumstance that results in individual psychological or behavioural deviations. Stress may be related to or exacerbated by many of the emotional and physical symptoms that occur frequently in the college community, such as headaches, fatigue, depression, anxiety, and the inability to cope. The cognitive process of the individual plays a significant role in determining whether he or she interpreted a specific event as stressful. The importance of this process is made clear by the fact that when confronted with those same potentially stress inducing situation, some person experience stress whereas others do not.

Perceived stress is an individual's feeling or a thought about the amount of stress they are under at a particular point in time or over a particular period of time. Perceived stress includes uncontrollability and unpredictability feelings about one's life. It measures how an individual feels about the over-all stressfulness of their life to deal with such stress and not the types or frequencies of stressful events which have happened to them.

\section{REVIEW OF LITERATURE}

There have been studies that show that empathy is predicted by age, gender (DiLalla, 2004; Endresen \& Olweus, 2001; Schieman \& Van Gundy, 2000) and education (Alligood, 2007; Spencer, 2004), where higher education, higher age and female gender are correlated with higher empathy levels. Stereotypically, females have a better perception of the thoughts and attitudes of others than males. A gender disparity in human empathy and its associated behaviours was proposed by Hoffman in 1977. He found, for example, that four out of five women report sharing friends' emotional distress more often than the average male does. The gender gap was significant in just a few of the publications examined and the differences were minimal.

Haque et al., 2018 conducted a cross sectional study among the medical students in Malaysia to assess their empathy levels and the results indicated that female students reported to have a statistically significantly higher score in empathy than the male students. A research study done by Courtright et al., 2005 examined the level of empathy among college students and compared the differences in gender across various scales that measured empathy and results indicated that females had higher level of empathy than males. Similar results were found in studies done by Hasan et. al, Williams et al. and Atan who compared the empathy levels among males and females and reported that females have a higher empathy than males (Atan, 2017; Hasan et al., 2013; Williams et al., 2014).

A research done by C. et al., 2017 on gender differences in perceived stress and coping strategies among college students showed that females had higher level of perceived stress; although the sample data did not provide enough evidence to show a significant gender difference in perceived stress among college students. In response to initial and repeated exposure to a laboratory stressor, Schmaus et al., 2008 explored gender disparities, as well as possible explanations for these differences. The results showed that, relative to men indicating sensitization, women might be more prone to repeated exposures to stress. A study done by Misigo on gender difference in perceived level of stress and coping strategies among university students in Kenya showed that females have a higher level of perceived stress and a significant difference was found between the stress mean scores of males and females (Misigo, 2015). A study done by Thawabieh and Qaisy to assess the level of stress experienced by university students reported that the students experienced a moderate amount of stress and the female students had more stress level than males (Thawabieh \& Qaisy, 2012). Thoits (1995) indicated that while it is believed that gender has an influence on the

(c) The International Journal of Indian Psychology, ISSN 2348-5396 (e)| ISSN: 2349-3429 (p) | 222 


\section{Empathy and Perceived Stress among College Students}

link between stress and the form of coping ability chosen, these findings are not always consistent. (Thoits, 1995).

Growing research indicates that various types of circumstances stress women and men. Men were more likely to list financial and work-related behaviours as causes of their stress, while women were more likely to list incidents related to family and health. Studies have found, however, that college students encounter stress related to financial issues, examination distress, rejection, relationship breakdown, depression, and low self-esteem feelings.

Empathy promotes abilities that help a person to handle stress. Studies show that when one can regulate their emotions, they are better able to relate others in positive ways. A study conducted by Hojat et al., 2009 found a substantial decrease in the medical students' empathy scores upon entry into their clinical years (after the third year). A result similar to this was found in a study done by Chen et al., 2007 who found that empathy scores of the students decreased at the end of the third year. A different trend was reported by Kozený et al., 2013: a continuous decrease in empathy scores during the first 4 years of medical study, followed by an increase during the following 2 years; however, the authors noted that in their clinical years (years 5-6), the average empathy scores of students were lower compared to students in their preclinical years (years 1-4). On the other hand, contradictory trends have been found in the previous researches regarding the stress level. A study done by Hasan et al., 2013 to assess the empathy level of medical students in Kuwait University Medical School and its relationship with sociodemographic factors, stress levels and personality showed that the levels of stress were found to be significantly and positively associated with empathy and higher levels of empathy were reported among female students. A study conducted by Wahjudi et al., 2019 to assess the relationship between empathy and stress among under graduate medical students suggested that there was no significant correlation among these variables, however, a sharp decrease in empathy decline among medical students upon entering their first clinical year was observed.

There have been studies which show that Asian culture can influence variations in the sentiment and empathy of people, including less reliance on non-verbal communication and assertiveness (Hong et al., 2012; Kataoka et al., 2009). When communicating with the environment, this also relates to stress and coping mechanisms; students from East Asian backgrounds appear to have internally targeted management techniques (e.g. used selfcontrol, waiting, embracing circumstance, and accountability), whereas those from Western backgrounds depend more on self-improving interpretive control (e.g., the use of optimistic reassessment in conjugation with external factors or environment) (Tweed et al., 2004).

Though many studies have been conducted on the relationship between empathy and stress levels but the studies are very less in India compared to other countries, and most of the studies done were on medical students who are only a specific part of the college population. To increase the understanding of this topic, even among the general college students (be it any field of study) the present study recruited a sample of undergraduate and post graduate college students from West Bengal and Karnataka in India to assess the relationship between their stress and empathy levels.

\section{METHODOLOGY}

The researchers had raised the following research questions which have been answered in the present study. 


\section{Empathy and Perceived Stress among College Students}

\section{Research Questions}

- Is there a significant relationship between cognitive empathy and perceived stress among college students?

- Is there a significant relationship between affective empathy and perceived stress among college students?

- Is there a significant gender difference in empathy among college students?

- Is there a significant gender difference in perceived stress among college students?

- In order to answer the above research questions, the following objectives had been developed and achieved in the present study.

\section{Objectives}

- To assess the relationship between cognitive empathy and perceived stress among college students.

- To assess the relationship between affective empathy and perceived stress among college students.

- To find out the gender difference in empathy among college students.

- To find out the gender difference in perceived stress among college students

- In order to achieve the above objectives, the following hypotheses had been generated and tested in this study.

\section{Hypotheses}

H1: There is a significant relationship between cognitive empathy and perceived stress among college students.

H2: There is a significant relationship between affective empathy and perceived stress among college students.

$\boldsymbol{H}_{0} 3$ : There is no significant gender difference in empathy among college students.

$\boldsymbol{H}_{04}$ : There is no significant gender difference in perceived stress among college students.

\section{Design of the Study}

A descriptive, non experimental research design was employed using quantitative approach to assess the relationship between empathy and perceived stress and to find out the gender difference with respect to empathy and perceived stress among college students in this present study.

\section{Sample}

The sample of the study comprised 214 students of age group 18-25 years, of which there were 107 boys and 107 girls studying in the colleges of the states of West Bengal and Karnataka in India, selected using non probability purposive sampling technique.

Tools used

\begin{tabular}{|l|l|l|l|l|}
\hline Variable & Tool & Developer & $\begin{array}{l}\text { Likert } \\
\text { Type }\end{array}$ & $\begin{array}{l}\text { Validity/ } \\
\text { Reliability }\end{array}$ \\
\hline Empathy & $\begin{array}{l}\text { Basic Empathy } \\
\text { Scale in Adults } \\
\text { (BES-A) }\end{array}$ & $\begin{array}{l}\text { Jolliffe and } \\
\text { Farrington (2006) }\end{array}$ & $\begin{array}{l}\text { 5-point } \\
\text { scale }\end{array}$ & $\begin{array}{l}\text { Internal } \\
\text { consistency } \\
(\alpha)-0.79 \text { to } \\
0.85\end{array}$ \\
\hline Perceived Stress & PSS-10 & Cohen et al. (1983) & $\begin{array}{l}\text { 5-point } \\
\text { scale }\end{array}$ & $\begin{array}{l}\text { Cronbach's } \\
\text { coefficient- } \\
\text { above .70 and } \\
\text { even upto .91 }\end{array}$ \\
\hline
\end{tabular}

(c) The International Journal of Indian Psychology, ISSN 2348-5396 (e)| ISSN: 2349-3429 (p) | 224 


\section{RESULTS AND DISCUSSION}

The data collected were scored, tabulated and also their descriptive statistics were calculated. In turn, the outcomes or results were interpreted hypothesis wise with the help of inferential statistics. The results obtained are presented as well as discussed as follows:

\section{H1: There is a significant relationship between cognitive empathy and perceived stress among college students.}

In order to test the above hypothesis, the mean scores obtained by the college students on perceived stress and cognitive empathy was computed apart from the computation of SD. Further, the significance of the relationship between cognitive empathy and perceived stress was computed using Pearson correlation test which yielded the following.

Table 1. Correlation between cognitive empathy and perceived stress among college students

\begin{tabular}{llllcc}
\hline & N & M & SD & r & p \\
\hline PSS & 214 & 20.43 & 6.30 & -.003 & 0.971 \\
CogET & & 34.20 & 4.13 & & \\
\hline Note: PSS Perceived Stress & CogET & Cognive &
\end{tabular}

Note: PSS = Perceived Stress, CogET= Cognitive Empathy

An analysis of the above table indicates that there were 214 college students. The mean score of the students for perceived stress was found to be 20.43, while that for cognitive empathy was 34.20. While in terms of standard deviation, the perceived stress had 6.30 and cognitive empathy had 4.13. It means the spread of scores away from the mean was apparently more for perceived stress suggesting that the variation was more for perceived stress among the college students. In order to see whether there was a significant relationship between cognitive empathy and perceived stress among the college students, the scores were subjected to Pearson correlation test, which yielded the person correlation coefficient ' $r$ ' as -.003, which was statistically insignificant at both the levels. Therefore, the alternative hypothesis was rejected which means that there was no significant relationship between these two variables. This can be supported by a study done by Wahjudi et al., 2019 who found no significant relationship between cognitive empathy and perceived stress among undergraduate medical students. Despite the fact that no association was found between cognitive empathy and perceived stress among the college students in this study, the researcher would like to highlight the fact that different stressors are interpreted and tackled differently by people who come from different cultures (Hashimoto et al., n.d.; Hong et al., 2012; Tweed et al., 2004). The researchers believe that since India is a diversified country and people from different cultures and different states study together, the way in which they manage stress, think and analyze different situations varies greatly. Thus, this could be a possible reason that no significant relationship was found between perceived stress and cognitive empathy among the college students.

\section{H2: There is a significant relationship between affective empathy and perceived stress among college students.}

In order to test the above hypothesis, the mean scores obtained by the college students on perceived stress and cognitive empathy was computed apart from the computation of SD. Further, the significance of the relationship between cognitive empathy and perceived stress was computed using Pearson correlation test which yielded the following. 
Empathy and Perceived Stress among College Students

Table 2. Correlation between affective empathy and perceived stress among college students

\begin{tabular}{llllcc}
\hline & $\mathbf{N}$ & $\mathbf{M}$ & SD & $\mathbf{r}$ & $\mathbf{p}$ \\
\hline PSS & 214 & 20.43 & 6.30 & $.287^{* *}$ & .01 \\
AFT & & 39.64 & 5.61 & & \\
\hline
\end{tabular}

Note: $P S S=$ Perceived Stress, AFT $=$ Affective Empathy, ${ }^{*} * p<0.01$

An analysis of the above table indicates that there were 214 college students. The mean score of the students for perceived stress was found to be 20.43, while that for affective empathy was 39.64. While in terms of standard deviation, the perceived stress had 6.30 and affective empathy had 5.61. It means the spread of scores away from the mean was apparently more for perceived stress suggesting that the variation was more for perceived stress among the college students. In order to see whether there was a significant relationship between affective empathy and perceived stress among the college students, the scores were subjected to Pearson correlation test, which yielded the person correlation coefficient ' $r$ ' as .287, which was statistically significant at 0.01 level. Therefore, the alternative hypothesis was accepted which means that there was a significant relationship between the affective empathy and perceived stress among college students. This finding is consistent with the previous studies which showed a significant relationship between these two variables (Chen et al., 2007; Hojat et al., 2009; Kozený et al., 2013). In this study a significant positive correlation was found between affective empathy and perceived stress and a similar result was also found in a study done by Hasan et al., 2013 in which the stress levels were found to be significantly and positively associated with empathy.

Ho3: There is no significant gender difference in empathy among college students.

In order to test the above hypothesis, the mean scores obtained by the boys and girls on empathy was computed apart from the computation of SD and SE. Further, the significance of the difference between mean scores of the two groups was computed using " $t$ " test which yielded the following.

Table 3. Independent Sample t test for the gender difference in empathy among college students

\begin{tabular}{llllllll}
\hline & N & M & SD & SE & Df & t & p \\
\hline Boys & 107 & 72.31 & 7.58 & .73 & & & \\
Girls & 107 & 75.37 & 7.69 & .74 & 212 & $2.938^{* *}$ & .01
\end{tabular}

Note: $* * \mathrm{p}<0.01$

An analysis of the above table indicates that there were 214 college students among which 107 were boys and 107 were girls. The mean score of the boys on empathy was found to be 72.31, while that for girls was 75.37. Although both the groups had high level of empathy, apparently, the girls had a higher level of empathy when compared to boys. While in terms of standard deviation, the boys had 7.58 and girls had 7.69. It means the spread of scores away from the mean was apparently more for girls suggesting that the variation was more for girls rather than boys. In order to test the obtained mean difference for their statistical significance, the scores were subjected to independent sample t test and it was found to be 2.938, which was statistically significant at 0.01 level. Therefore, the null hypothesis was rejected. It means that there was a significant gender difference in empathy among college students. These findings can be supported by the results obtained in the previous studies in 
which females were reported to have higher empathy levels (Atan, 2017; Courtright et al., 2005; Haque et al., 2018; Hasan et al., 2013; Williams et al., 2014). These findings could perhaps be explained by the fact that females usually may be more motivated to be empathic and thus may show higher empathy levels (Klein \& Hodges, 2001) and stereotypically also it is believed that females have a better perception of the thoughts and attitudes of others than males.

\section{H04: There is no significant gender difference in perceived stress among college students.}

In order to test the above hypothesis, the mean scores obtained by the boys and girls on perceived stress was computed apart from the computation of SD and SE. Further, the significance of the difference between mean scores of the two groups was computed using " $t$ " test which yielded the following.

Table 4. Independent Sample t test for the gender difference in perceived stress among college students

\begin{tabular}{llllllll}
\hline & $\mathbf{N}$ & $\mathbf{M}$ & SD & SE & df & t & p \\
Boys & 107 & 19.77 & 6.17 & .597 & & & \\
Girls & 107 & 21.08 & 6.37 & .616 & 212 & 1.537 & .445 \\
\hline
\end{tabular}

An analysis of the above table indicates that there were 214 college students among which 107 were boys and 107 were girls. The mean score of the boys on perceived stress was found to be 19.77, while that for girls was 21.08. There was a minimal difference of mean scores in favor of girls which indicates that they had a higher perceived stress than boys. In terms of standard deviation, the boys scored 6.17 and girls scored 6.37 suggesting that the scores of girls were spread more away from the mean scores than that of the boys. In order to test the obtained mean difference for their statistical significance, the scores were subjected to independent sample t test and it was found to be 1.537 , which was not significant at both the levels. So, the null hypothesis was accepted. It means that there was no significant gender difference in perceived stress among college students. So, it can be said that the gender had nothing to do with perceived stress among the college students. This finding is consistent with the previous studies which showed that females experience a higher level of perceived stress than males (C. et al., 2017; Misigo, 2015; Schmaus et al., 2008; Thawabieh \& Qaisy, 2012). Although the females showed a higher level of perceived stress, the sample data did not provide enough evidence to show a significant gender difference in perceived stress among college students and a similar result was also obtained by C. et al., 2017 who reported that the females had high perceived stress levels but no significant difference in perceived stress was found with respect to gender. This can be attributed to the fact that even though it is believed that gender has an influence on the link between stress and the form of coping ability chosen, these findings are not always consistent (Thoits, 1995).

\section{CONCLUSION}

- There was no significant relationship between the cognitive empathy and perceived stress among the college students.

- There was a significant relationship between the affective empathy and perceived stress among the college students and this correlation was found to be positive. 


\section{Empathy and Perceived Stress among College Students}

- There was a significant gender difference in empathy among college students and girls had higher empathy than boys.

- No significant gender difference was found with respect to perceived stress among college students; although the girls showed higher perceived stress levels than boys but the difference in their perceived stress levels was minimal.

\section{Limitations}

This study has the following limitations:

- This study covers only the college students as a sample and hence the results may not hold good for the other parts or segments of the population.

- In this study the students of age group 18-25 were only considered; thus, the findings may not be applicable to students of other age groups.

- The sample students were from West Bengal and Karnataka and thus, the findings may not be true for the students from other states in India.

- This study was based on a self-report questionnaire, so there might be a response bias, which cannot be ignored.

- This study was based on a correlational research design, so no conclusion can be drawn about the causal relationship between empathy and perceived stress.

- The perceived stress scale used measures the stress levels of an individual relating to the previous month, so nothing can be concluded about the present stress levels of the college students and results regarding their stress levels might be temporary.

\section{Implications}

Although the total mean scores of empathies for both male and female college students was high, action should be taken from the college administration to ensure consistency in the development of empathetic skills of the students until they start working. Improvements in curriculum development and teaching methods should be done in order to ensure that these aspects help the students to become more empathetic in the near future.

Also, there is also a need for the college administration to set up stress management programmes and increase the counselling facilities and services for the students to help them cope up with their stress levels. Further, the parents and family members may also play an essential role in order to minimise the stress level of the college students by extending their support. Although, it is also important to mention that some amount of stress is important for the college students to prepare themselves to be competitive and push them to their fullest potential.

\section{Suggestions for future research}

Whereas some contribution to the existing literature has been made by this study, these findings are only limited to a particular age group and to the college students belonging from some particular states in India. Further research is needed on other samples from other states and countries as well. Studies should also be conducted in secondary schools and students beyond the age group of 18-25 years to further determine whether there is support for the findings of this research or not. In future studies, other measurements can be used to determine the different aspects of empathy and stress levels as well as other sociodemographic variables can be compared to determine the significant difference in the empathy and perceived stress levels. A mixed approach of qualitative and quantitative methods can be used which will help to explore more possibilities in the study. 
REFERENCES

Atan, T. (2017). Empathy Levels of University Students Who Do and Not Do Sports. Universal Journal of Educational Research, 5(3), 500-503. https://doi.org/10.13189/ujer.2017.050322

Batson, C. D. (1995). Prosocial motivation: Why do we help others. Advanced Social Psychology, 333-381.

Brougham, R., Zail, C., Mendoza, C., \& Miller, J. (2009). Stress, Sex Differences, and Coping Strategies Among College Students. Current Psychology, 28, 85-97. https://doi.org/10.1007/s12144-009-9047-0

C., A., P., D., V.P., J., Dhandapani, P., \& D., R. (2017). Gender Differences in Perceived Stress levels and Coping Strategies among College Students. The International Journal of Indian Psychology, 4. https://doi.org/10.25215/0404.103

Campbell, R. G., \& Babrow, A. S. (2004). The Role of Empathy in Responses to Persuasive Risk Communication: Overcoming Resistance to HIV Prevention Messages. Health Communication, 16(2), 159-182. https://doi.org/10.1207/S15327027HC1602_2

Chao, R. C. (2012). Managing perceived stress among college students: The roles of social support and dysfunctional coping. Journal of College Counseling, 15(1), 5-21.

Chao, R. C.-L. (2012). Managing Perceived Stress Among College Students: The Roles of Social Support and Dysfunctional Coping. Journal of College Counseling, 15(1), 521. https://doi.org/10.1002/j.2161-1882.2012.00002.x

Chen, D., Lew, R., Hershman, W., \& Orlander, J. (2007). A cross-sectional measurement of medical student empathy. Journal of General Internal Medicine, 22(10), 1434-1438.

Courtright, K., Mackey, D., \& Packard, S. (2005). Empathy among college students and criminal justice majors: Identifying predispositional traits and the role of education. Journal of Criminal Justice Education, 16, 125-144. https://doi.org/10.1080/1051125042000333514

Davis, M. H. (1996). Empathy: A social psychological approach. Boulder, Colorado. Westview Press, Inc.

Davis, Mark H. (1983). Measuring individual differences in empathy: Evidence for a multidimensional approach. Journal of Personality and Social Psychology, 44(1), 113-126. https://doi.org/10.1037/0022-3514.44.1.113

Duan, C., \& Hill, C. E. (1996). The current state of empathy research. Journal of Counseling Psychology, 43(3), 261.

Haque, M., Sa, B., Majumder, M. A., Islam, M., Othman, N., Lutfi, S., Kibria, G. M., Salam, A., Ismail, M. H., \& Abdullah, S. (2018). Empathy among Undergraduate Medical Students: A Cross-Sectional Study in One Malaysian Public Medical School. Annals of African Medicine, 17, 183. https://doi.org/10.4103/aam.aam_57_17

Hasan, S., Al-Sharqawi, N., Dashti, F., AbdulAziz, M., Abdullah, A., Shukkur, M., Bouhaimed, M., \& Thalib, L. (2013). Level of Empathy among Medical Students in Kuwait University, Kuwait. Medical Principles and Practice, 22(4), 385-389. https://doi.org/10.1159/000348300

Hashimoto, T., Mojaverian, T., \& Kim, H. (n.d.). Culture, Interpersonal Stress, and Psychological Distress. Sage Publications.

https://www.academia.edu/11022541/Culture_Interpersonal_Stress_and_Psychologic al_Distress

Hogan, J., Carlson, J., \& Dua, J. (2002). Stressors and Stress Reactions Among University Personnel. International Journal of Stress Management, 9, 289-310. https://doi.org/10.1023/A:1019982316327 


\section{Empathy and Perceived Stress among College Students}

Hojat, M., Vergare, M. J., Maxwell, K., Brainard, G., Herrine, S. K., Isenberg, G. A., Veloski, J., \& Gonnella, J. S. (2009). The devil is in the third year: A longitudinal study of erosion of empathy in medical school. Academic Medicine, 84(9), 1182-1191.

Hong, M., Lee, W. H., Park, J. H., Yoon, T. Y., Moon, D. S., Lee, S. M., \& Bahn, G. H. (2012). Changes of empathy in medical college and medical school students: 1-year follow up study. BMC Medical Education, 12(1), 122.

Kataoka, H. U., Koide, N., Ochi, K., Hojat, M., \& Gonnella, J. S. (2009). Measurement of empathy among Japanese medical students: Psychometrics and score differences by gender and level of medical education. Academic Medicine, 84(9), 1192-1197.

Kozený, J., TIŠANSKÁ, L., \& Hoeschl, C. (2013). Assesing Empathy among Czech Medical Students: A Cross-Sectional Study. Ceskoslovenská Psychologie, 57(3).

Klein, K. J., \& Hodges, S. D. (2001a). Gender differences, motivation, and empathic accuracy: When it pays to understand. Personality and Social Psychology Bulletin, 27(6), 720-730.

Misigo, B. L. (2015). Gender Difference in The Perceived Level of Stress and Coping Strategies Among University Students in Kenya: A Case of Public Universities. Open Access, 1(4), 9.

Ross, S. E., Niebling, B. C., \& Heckert, T. M. (1999). Sources of stress among college students. Social Psychology, 61(5), 841-846.

Schmaus, B. J., Laubmeier, K. K., Boquiren, V. M., Herzer, M., \& Zakowski, S. G. (2008). Gender and stress: Differential psychophysiological reactivity to stress reexposure in the laboratory. International Journal of Psychophysiology: Official Journal of the International Organization of Psychophysiology, 69(2), 101-106. https://doi.org/10.1016/j.ijpsycho.2008.03.006

Thawabieh, A., \& Qaisy, L. (n.d.). Assessing Stress among University Students. American International Journal of Contemporary Research.

Thoits, P. A. (1995). Stress, Coping, and Social Support Processes: Where Are We? What Next? Journal of Health and Social Behavior, 53-79. https://doi.org/10.2307/2626957

Tweed, R. G., White, K., \& Lehman, D. R. (2004). Culture, stress, and coping: Internallyand externally-targeted control strategies of European Canadians, East Asian Canadians, and Japanese. Journal of Cross-Cultural Psychology, 35(6), 652-668.

Wahjudi, J. W., Findyartini, A., \& Kaligis, F. (2019). The relationship between empathy and stress: A cross-sectional study among undergraduate medical students. Korean Journal of Medical Education, 31(3), 215-226. https://doi.org/10.3946/kjme.2019.132

Williams, B., Brown, T., Boyle, M., McKenna, L., Palermo, C., \& Etherington, J. (2014). Levels of empathy in undergraduate emergency health, nursing, and midwifery students: A longitudinal study. Advances in Medical Education and Practice, 5, 299 306. https://doi.org/10.2147/AMEP.S66681

\section{Acknowledgement}

The author(s) appreciates all those who participated in the study and helped to facilitate the research process.

\section{Conflict of Interest}

The author(s) declared no conflict of interest. 


\section{Empathy and Perceived Stress among College Students}

How to cite this article: Gupta K. \& Kiran NC (2021). Empathy and Perceived Stress among College Students. International Journal of Indian Psychology, 9(2), 220-231. DIP:18.01.027.20210902, DOI:10.25215/0902.027 